\title{
Conduta Expectante para Gravidez Tubária Íntegra.
}

\author{
Expectant Management for (Tubal) Ectopic Pregnancy
}

Kyung Koo Han, Julio Elito Junior, Luiz Camano.

\begin{abstract}
RESUMO
Objetivo: avaliar os parâmetros de inclusão empregados na seleção dos casos de gravidez tubária integra para a conduta expectante e avaliar os resultados.

Métodos: foi realizado estudo prospectivo em 70 pacientes com diagnóstico de gravidez tubária integra, em que se optou pela conduta expectante. Os principais critérios de inclusão foram: o diâmetro transversal da massa tubária igual ou inferior a $5,0 \mathrm{~cm}$, titulos de beta-hCG (fração beta do hormônio gonadotrópico coriônico) em declinio de pelo menos $2 \%$ num intervalo de 48 horas, estabilidade hemodinâmica, desejo de gravidez futura e consentimento escrito para participar do estudo. Todas as pacientes foram observadas em regime de internação hospitalar e todas receberam alta hospitalar após se constatar o declinio dos títulos de betahCG e foram acompanhadas ambulatorialmente com dosagens semanais dos titulos até que estes atingissem valores inferiores a $5 \mathrm{mUI} / \mathrm{ml}$, situação que consideramos como sucesso.

Resultados: das 70 pacientes submetidas à conduta expectante, apenas uma precisou de intervenção cirúrgica por ruptura tubária. Os valores iniciais dos titulos de beta-hCG das pacientes variaram de $27 \mathrm{mUI} / \mathrm{ml}$ a $41.000 \mathrm{mUI} / \mathrm{ml}$. A média do diâmetro da massa tubária foi 2,9 cm. Liquido livre na cavidade peritoneal foi observado em 50 pacientes, sendo a quantidade pequena em 26, moderada em 16 e grande em 8 casos. O aspecto ultra-sonográfico característico de hematossalpinge ocorreu em 58 pacientes e o anel tubário, em 12. Ao Doppler colorido, 52 foram classificadas como de baixo risco e 18, de médio risco.

Conclusão: a conduta expectante pode ser empregada com segurança nos casos que respeitarem os critérios de inclusão, sendo o indice de sucesso de 98,6\%.
\end{abstract}

PALAVRAS-CHAVE: Gravidez ectópica. Gravidez tubária integra. Conduta expectante. Gonadotrofinas coriônicas.

\section{Introdução}

Desde o emprego inicial da conduta expectante para a gravidez ectópica (GE) em 1955, com Lund ${ }^{1}$, esta vem sendo aplicada como uma alternativa terapêtica em alguns casos selecionados. Ao selecionarmos pacientes com gravidez tubária íntegra (GTI) para conduta expectante, evitam-se riscos anestésico-cirúrgicos e os custos são menores. Nos EUA, para cada paciente tratada cirurgicamente gastam-se

Disciplina de Obstetrícia da Escola Paulista de Medicina Universidade Federal de São Paulo.

Correspondência:

Kyung Koo Han

R. Raul Pompéia 199, Apto 113 - Pompéia

05025-010 - São Paulo - SP

Tel/Fax: (11) 3862-8177
7.972,00 dólares e quando se opta pelo tratamento clínico (uso de quimioterápico ou a conduta expectante) economiza-se até $24 \%$ deste valor. Isto representa uma economia de 28 milhões de dólares por $\mathrm{ano}^{2}$.

Diversos autores já indicaram esta conduta nos casos específicos de GTI com indices de sucesso que oscilam entre 47,7 e $92,0 \% \%^{3,45,6,7}$. Os autores citados utilizaram os seguintes critérios de inclusão para adotar a conduta expectante: estabilidade hemodinâmica, diâmetro transversal da massa tubária igual ou menor a $4,0 \mathrm{~cm}$, queda dos títulos de beta-hCG num intervalo de 48 horas e desejo de gravidez futura.

O objetivo deste trabalho é avaliar os parâmetros de inclusão empregados na seleção dos casos de gravidez tubária întegra para a conduta expectante e avaliar os resultados. 


\section{Pacientes e Métodos}

Este estudo prospectivo foi realizado na Disciplina de Obstetrícia da Universidade Federal de São Paulo - Escola Paulista de Medicina, no período de fevereiro de 1997 a maio de 1998. Neste período foram atendidas no total 120 pacientes com diagnóstico de gravidez tubária e apenas 74 pacientes com a tuba integra. Deste grupo apenas 70 foram incluídas no trabalho, pois preenchiam todos os critérios de inclusão. As restantes foram submetidas a salpingectomia total ipsilateral à gravidez ectópica (GE). Para o diagnóstico precoce de gravidez tubária integra (GTI), valorizamos os dados da história clínica e do exame físico, juntamente com os exames subsidiários: dosagem sérica da fração beta do hormônio gonadotrópico coriônico (beta-hCG) e a ultra-sonografia (US) transvaginal.

Firmado o diagnóstico de GTI, seguimos alguns critérios para decidir pela conduta expectante. Os critérios foram: estabilidade hemodinâmica, queda dos títulos de beta-hCG num intervalo de 48 horas, diâmetro da massa tubária menor ou igual a $5,0 \mathrm{~cm}$, desejo manifesto de gravidez futura pela paciente e concordância em participar do estudo, através de consentimento escrito, em documento formulado e aprovado pela Comissão de Ética Médica da UNIFESP-EPM. Foram excluídas da conduta expectante as pacientes com necessidade de hemotransfusão, as que apresentaram Doppler colorido de elevado risco na massa tubária ou presença do embrião vivo à luz da US transvaginal e as que não apresentaram declínio dos títulos de beta-hCG num intervalo de 48 horas. Nestes casos as pacientes foram informadas da possibilidade de se realizar o tratamento medicamentoso com metotrexato (MTX) em dose única $\left(50 \mathrm{mg} / \mathrm{m}^{2}\right.$ de superfície corporal) por via intramuscular ${ }^{8}$ ou cirurgia (laparotomia ou laparoscopia).

As dosagens de beta-hCG foram realizadas pelo método imunoenzimométrico (AIA-Pack bhCG, Tosoh Medics, Inc.). A US foi realizada com equipamento ATL UM-9 (Bothel, Wash) com transdutor de banda larga de $5-9 \mathrm{mHz}$ para via transvaginal. Após minucioso exame em escalas de cinza, utilizamos o Doppler colorido para o mapeamento vascular do útero, ovários e eventuais massas tubárias, seguindo-se por último a análise espectral dos fluxos encontrados nos anexos e, quando necessário, da região periendometrial.

As massas tubárias foram caracterizadas conforme o seu aspecto ao US em: hematossalpinge, anel tubário e saco gestacional com embrião vivo. A imagem patognomônica da GTI é a presença de saco gestacional ectópico, o qual contém o embrião e este apresenta batimentos cardíacos. Quanto ao anel tubário, podemos encontrar na US as seguintes situações: saco gestacional que pode apresentar embrião, porém sem atividade cardiaca, saco gestacional contendo apenas a vesícula vitelínica e saco gestacional vazio, sendo que este se apresenta hiperecogênico e tem forma de anel, com seu centro anecóico.

A hematossalpinge tem o seguinte aspecto à ecografia: presença de massa sólida, com a ecogenicidade complexa, apresentando áreas hiper e hipoecóicas irregulares.

$\mathrm{Na}$ avaliação US pode-se determinar o diâmetro transversal da massa tubária, sendo este um dos principais critérios de inclusão no grupo para a conduta expectante. Desta forma, em nosso estudo todas as pacientes apresentavam o diâmetro transversal da massa tubária de até $5,0 \mathrm{~cm}$.

A quantidade de liquido livre na cavidade peritoneal foi observada e seu volume calculado conforme o trabalho de Nyberg et al. ${ }^{9}$, com algumas modificações por nós realizadas. Classificamos como discreta (líquido apenas no fundo-de-saco de Douglas), moderada (estendendo-se para região perianexial ipsilateral) e acentuada (extensão para regiões peri/supra-uterina e anexiais).

Na US com Doppler colorido caracterizou-se como fluxo trofoblástico a presença de fluxo arterial de baixa resistência ao redor da massa tubária, com indice de resistência (IR) menor que 0,45. Como conseqüência dos estudos dopplervelocimétricos os casos foram classificados em três grupos: elevado, médio e baixo risco. O de elevado risco foi caracterizado por fluxo trofoblástico em mais de 2/3 da massa tubária; de médio risco, quando comprometeu de $1 / 3$ a 2/3 do anel tubário, como descreveram Atri et al. ${ }^{10}$; classificamos como de baixo risco as seguintes situações: fluxos que acometiam menos de $1 / 3$ da massa tubária; ausência de vascularização ao redor da massa e presença de fluxos arteriais de resistência aumentada (IR maior que 0,5); fluxos com morfologia bizarra quando se apresentavam com importante alargamento espectral, com tempos de aceleração longos e diástoles ausentes ou negativas.

Quando internamos as pacientes para a conduta expectante, realizamos de imediato a dosagem de beta-hCG sérico. Dependendo deste título inicial, dividimos em dois grupos:

- beta-hCG inicial $\geq 5.000 \mathrm{mUI} / \mathrm{ml}$ : neste grupo, além da dosagem inicial do beta-hCG, realizamos mais duas medidas, com intervalo de 48 horas. Assim, tivemos três avaliações deste hormônio apuradas no $1^{\circ}, 3^{\circ}$ e no $5^{\circ}$ dia da internação. 
Ocorrendo, entre estes valores, declínio dos títulos de beta-hCG entre o $1^{\circ}$ e $3^{\circ}$ dia e entre o $3^{\circ}$ e $5^{\circ}$ dias as pacientes recebiam alta hospitalar e retornavam semanalmente ao ambulatório para dosagem dos títulos de beta-hCG até que estes atingissem niveis pré-gravídicos.

- beta-hCG inicial <5.000 mUI/ml: neste grupo, realizamos apenas duas medidas dos títulos de beta-hCG, ou seja, a inicial na admissão e 48 horas depois. Ocorrendo declínio dos títulos de beta-hCG entre estes dias, as pacientes recebiam alta hospitalar e retornavam semanalmente ao ambulatório para quantificar os títulos de betahCG, até que estes atingissem niveis prégravídicos.

Durante a internação, foi realizado acompanhamento clínico cuidadoso e US transvaginal, principalmente nos casos que apresentaram dor abdominal. O critério de insucesso na conduta expectante da GTI baseouse na ruptura tubária. Após a alta hospitalar, as pacientes foram seguidas semanalmente em ambulatório realizando-se avaliações clínicas, US e dosagens de beta-hCG até os títulos atingirem os valores negativos, situações consideradas como sucesso da conduta. As relações sexuais foram liberadas após o retorno dos valores de beta-hCG a niveis pré-gravídicos. Orientava-se então o uso de anticoncepcional oral ou de método de barreira, por um período de 3 meses aproximadamente, pois neste prazo, geralmente, ocorre o desaparecimento da imagem ultra-sonográfica da massa tubária. Como controle, foi realizada a histerossalpingografia (HSG) cerca de 3 meses após o desaparecimento da massa tubária à luz da US transvaginal. Posteriormente, as pacientes foram reavaliadas semestralmente, com anotação de nova gestação. Na ocorrência de nova gestação, as pacientes eram acompanhadas de forma personalizada pelo autor ao longo da gestação até o parto.

\section{Resultados}

De um grupo de 70 pacientes com diagnóstico de GTI, obtivemos sucesso em 69 delas com a conduta expectante e uma paciente precisou ser submetida a laparotomia por ruptura tubária. No nosso trabalho verificamos que a maioria dos casos (52,8\%) apresentava mais de 11 semanas de gestação, no momento do diagnóstico da doença.

Correlacionamos na Tabela 1 a presença de líquido livre com o sucesso e o insucesso da conduta e verificamos que $71,5 \%$ dos casos apresentaram liquido livre na cavidade peritoneal.
Tabela 1 - Presença de quantidade de líquido livre na cavidade peritoneal pela US transvaginal, em 70 casos de GTI em que se adotou conduta expectante.

\begin{tabular}{lrlr}
\hline $\begin{array}{l}\text { Quantidade } \\
\text { líquido livre }\end{array}$ & $\begin{array}{c}\text { Sucesso } \\
\mathbf{n}\end{array}$ & $\begin{array}{c}\text { Insucesso } \\
\mathbf{n}\end{array}$ & $\%$ \\
\hline Ausente & $20(28,5 \%)$ & 0 & 28,5 \\
Discreta & $26(37,2 \%)$ & 0 & 37,2 \\
Moderada & $15(21,5 \%)$ & $1(1,4 \%)$ & 22,9 \\
Acentuada & $8(11,4 \%)$ & 0 & 11,4 \\
Total & $69(98,6 \%)$ & $1(1,4 \%)$ & 100,0 \\
\hline
\end{tabular}

Na Tabela 2 podemos notar que, do grupo que culminou com sucesso, 57 pacientes $(81,5 \%)$ apresentavam hematossalpinge e 12 pacientes $(17,1 \%)$ apresentavam anel tubário.

Tabela 2 - Aspecto da massa tubária apurada pela US, em 70 casos de GTI em que se adotou conduta expectante.

\begin{tabular}{lclr}
\hline $\begin{array}{l}\text { Aspecto Ultra- } \\
\text { Sonográfico }\end{array}$ & $\begin{array}{c}\text { Sucesso } \\
\mathbf{n}\end{array}$ & $\begin{array}{c}\text { Insucesso } \\
\mathbf{n}\end{array}$ & $\%$ \\
\hline Hematossalpinge & $57(81,5 \%)$ & $1(1,4 \%)$ & 82,9 \\
Anel tubário & $12(17,1 \%)$ & 0 & 17,1 \\
Total & $69(98,6 \%)$ & $1(1,4 \%)$ & 100,0 \\
\hline
\end{tabular}

$\mathrm{Na}$ Tabela 3 correlacionamos Doppler colorido com o resultado da conduta. O Doppler de elevado risco não foi observado em nenhum caso. Isto ocorreu porque as pacientes que apresentavam Doppler de elevado risco foram excluídas do nosso trabalho, já que com este parâmetro desfavorável os outros critérios de inclusão também não seriam favoráveis. Das 70 pacientes, 18 (25,7\%), apresentaram Doppler de médio risco (quando verificamos que o fluxo trofoblástico acomete até $2 / 3$ da circunferência da tuba à luz da US transvaginal) e 52 (74,3\%) de baixo risco (quando o acometimento é menor que $1 / 3$ da tuba).

Tabela 3 - Aspecto da massa tubária pela dopplervelocimetria colorida, em 70 casos de GTI em que se adotou conduta expectante.

\begin{tabular}{lclr}
\hline Aspecto do Doppler & $\begin{array}{c}\text { Sucesso } \\
\mathbf{n}\end{array}$ & \multicolumn{1}{c}{$\begin{array}{c}\text { Insucesso } \\
\mathbf{n}\end{array}$} & \% \\
\hline Baixo Risco & $52(74,3 \%)$ & 0 & 74,3 \\
Médio Risco & $17(24,3 \%)$ & $1(1,4 \%)$ & 25,7 \\
Alto Risco & 0 & 0 & 0,0 \\
Total & $69(98,6 \%)$ & $1(1,4 \%)$ & 100,0 \\
\hline
\end{tabular}

A HSG foi efetuada em $24(34,7 \%)$ das 69 pacientes. Foi excluído 1 caso $(1,4 \%)$ que evoluiu 
para laparotomia, por insucesso da conduta, (vale lembrar que esta paciente era portadora de leiomioma uterino), e outras 45 se recusaram a realizar o exame. Das 24 pacientes que se submeteram ao exame, em $20(83,2 \%)$ foi detectada tuba pérvia ipsilateral à GE e $4(16,8 \%)$ mostraram obstrução; já a tuba contralateral apresentava-se pérvia em $100 \%$ dos casos.

Observando a Tabela 4, podemos perceber que a grande maioria das pacientes apresentou a variação de beta-hCG superior a $10 \%$ no intervalo de 48 horas iniciais $(85,7 \%$ dos casos) da internação. Salientamos que a média da variação no nosso estudo foi de 35,7 $\pm 23,25 \%$.

Dos casos incluídos no presente estudo, entre 7 pacientes desejosas de engravidar, cinco $(71,4 \%)$ o conseguiram, todas submetidas ao parto vaginal. Dentro da nossa população de estudo, estas

cinco pacientes representam $7,1 \%$ do total de casos. Na Tabela 5 apresentamos a relação entre o diâmetro transversal da tuba e o beta-hCG encontrado.

Tabela 4 - Declínio dos títulos de beta-hCG entre o primeiro e o terceiro dia nos 70 casos de GTI em que se adotou conduta expectante.

\begin{tabular}{ccc}
\hline $\begin{array}{c}\text { Variação (\%) } \\
\text { beta-hCG }\end{array}$ & $\mathbf{n}$ & $\%$ \\
\hline$<10$ & 10 & 14,3 \\
$10-20$ & 13 & 18,6 \\
$20-30$ & 10 & 14,3 \\
$30-40$ & 12 & 17,1 \\
$\geq 40$ & 25 & 35,7 \\
Total & 70 & 100,0 \\
\hline Média de porcentagem de declínio $=35,7 \pm 23,25 \%$ &
\end{tabular}

Tabela 5 - Correlação entre o diâmetro máximo da massa tubária apurada pela US com os valores iniciais de beta-hCG em 70 casos de GTI em que se adotou conduta expectante.

\begin{tabular}{lccccrr}
\hline $\begin{array}{c}\text { Diâmetro da massa } \\
(\mathbf{c m})\end{array}$ & $\begin{array}{c}\text { Beta-hCG } \\
<\mathbf{5 0 0 0}\end{array}$ & $\%$ & $\begin{array}{c}\text { Beta-hCG } \\
\mathbf{5 0 0 0}\end{array}$ & $\%$ & Valor Total & $\%$ \\
\hline$\leq 3,0$ & 43 & 61,4 & 6 & 8,6 & 49 & 70,0 \\
$3,0-4,0$ & 14 & 20,0 & 3 & 4,3 & 17 & 24,3 \\
$4,0-5,0$ & 1 & 1,4 & 3 & 4,3 & 4 & 5,7 \\
Total & 58 & 82,8 & 12 & 17,2 & 70 & 100,0 \\
\hline
\end{tabular}

Média da massa tubária $=2,88 \pm 0,69 \mathrm{~cm}$.

Para análise destes valores, utilizamos o teste "t" de Student (Tabela 6 ) para grupos independentes ${ }^{11}$ a fim de confrontar os valores dos diâmetros da tuba uterina entre os grupos com os títulos de beta-hCG $<5.000 \mathrm{mUI} / \mathrm{ml} \mathrm{e} \geq 5.000 \mathrm{mUI} /$ ml. Fixou-se em 0,05 ou $5 \%(p \leq 0,05)$ o nivel de significância.

\section{Discussão}

O diagnóstico precoce de GTI nem sempre é feito com facilidade e a paciente pode permanecer um período prolongado até que o diagnóstico definitivo seja feito. Em nosso estudo a maioria dos casos $(52,8 \%)$ apresentavam mais de 11 semanas de atraso menstrual. Nestes casos podemos inferir que a paciente passou por uma fase de ascenção dos títulos de beta-hCG, manutenção e, finalmente, declínio dos níveis deste hormônio.

O parâmetro importante a ser considerado é a presença de líquido livre na cavidade peritoneal. Pellerito et al. ${ }^{12}$ relataram a presença de líquido livre em 30\% dos casos de GE íntegra. Observamos também na maioria das nossas pacientes a presença de líquido em pequena quantidade nas assintomáticas e estáveis hemodinamicamente. Estas observações e os dados da literatura ${ }^{9,12}$ fizeram com que pudéssemos manter a conduta expectante e não nos alarmássemos perante a presença de líquido livre na cavidade peritoneal exceto na vigência de quantidade apreciável ou instabilidade hemodinâmica. A análise dessa variável deve sempre ser acompanhada de dados clínicos da paciente, como a análise da cor das mucosas, o pulso e a pressão arterial.

Observamos que os casos de GTI em involução inicialmente apresentam-se como anel tubário. Posteriormente ocorre o descolamento do trofoblasto de sua área de implantação, formandose o hematossalpinge devido à formação de coleção hemática. Por isso, neste estudo a maioria dos casos apresentavam-se como hematossalpinge, demonstrando desta forma a involução da GTI. 
Tabela 6 - Pacientes com gravidez tubária íntegra, segundo o diâmetro da tuba $(\mathrm{cm})$ e os valores de beta-hCG $<5.000 \mathrm{mUl} / \mathrm{ml}$ ou beta-hCG $\geq 5.000 \mathrm{mUl} / \mathrm{ml}$.

\begin{tabular}{|c|c|c|}
\hline \multicolumn{3}{|c|}{ DIÂMETRO } \\
\hline \multicolumn{2}{|c|}{$<5000 \mathrm{mUl} / \mathrm{ml}$} & \multirow{2}{*}{$\frac{\geq 5000 \mathrm{mUl} / \mathrm{ml}}{2,8}$} \\
\hline 3,5 & 2 & \\
\hline 3,8 & 2,2 & 2,5 \\
\hline 4 & 2,4 & 4,2 \\
\hline 4,2 & 2,5 & 2,5 \\
\hline 2,9 & 2,6 & 2,7 \\
\hline 2,9 & 2,8 & 4,6 \\
\hline 2,8 & 3,8 & 5 \\
\hline 2,5 & 2,6 & 2,5 \\
\hline 2,1 & 2,3 & 2,9 \\
\hline 1,8 & 2,4 & 3,7 \\
\hline 2 & 2,2 & 3,8 \\
\hline 2,7 & 2,8 & 3,8 \\
\hline 2,6 & 2,2 & \\
\hline 2,4 & 2,5 & \\
\hline 3,4 & 3,5 & \\
\hline 2,4 & 2,7 & \\
\hline 2,8 & 3,5 & \\
\hline 3,7 & 3,7 & \\
\hline 2,3 & 3,6 & \\
\hline 2,3 & 3,4 & \\
\hline 3,5 & 2,3 & \\
\hline 3,5 & 2,8 & \\
\hline 2,9 & 2,7 & \\
\hline 2,5 & 2,6 & \\
\hline 2,1 & 2,5 & \\
\hline 2,2 & 2,4 & \\
\hline 2,4 & 2,9 & \\
\hline 2,1 & 3,5 & \\
\hline 1,8 & 3,2 & \\
\hline Média & 2,78 & 3,44 \\
\hline DP & 0,60 & 0,87 \\
\hline
\end{tabular}

Teste " $\mathrm{t}$ " independente

$t_{\text {calc }}=-3,23^{*} \quad t_{\text {crit }}=1,99$

$<5.000<\geq 5.000$

*significante

O Doppler colorido é importante por diagnosticar os casos de baixo risco, o que tranqüiliza o médico e a paciente, enquanto se aguarda o resultado da segunda dosagem de betahCG. Consideramos o Doppler exame primordial e parâmetro de auxílio inegável no manejo das pacientes sob conduta expectante. Acreditamos todavia que a associação de outros parâmetros enriquece e nos oferece dados mais fidedignos para orientação da conduta.

Uma pergunta emergente no nosso trabalho era se haveria correlação entre o tamanho da massa tubária, apurado pela US transvaginal, e o valor de beta-hCG plasmática quando da admissão. Observamos que, das 58 pacientes com os niveis de beta-hCG inferiores a $5.000 \mathrm{mUI} / \mathrm{ml}$, a maioria $(61,4 \%)$ apresentava os diâmetros das massas menores ou iguais a $3,0 \mathrm{~cm}$. Das 12 pacientes com os niveis iniciais de beta-hCG superiores a 5.000 $\mathrm{mUI} / \mathrm{ml}$, metade apresentava diâmetros da massa tubária maiores ou iguais a $3,0 \mathrm{~cm}$. Portanto, quanto menor a massa tubária, menores foram os valores de beta-hCG encontrados. Segundo os autores que adotaram conduta expectante, um dos critérios mais importantes para avaliar a evolução é o declínio dos títulos de beta-hCG entre o primeiro dia e o terceiro dia (48 horas de intervalo). Se este declínio, em porcentagem, superar os $2 \%$, provavelmente haverá involução espontânea ${ }^{6}$.

A permeabilidade tubária após o emprego da conduta expectante é descrita como ocorrendo em 50 a $100 \%$ dos casos, com uma média de $76 \%$. A conduta expectante, portanto, pode preservar a permeabilidade tubária. No nosso estudo encontramos a tuba permeável após a conduta expectante em $83,2 \%$ dos casos. Uma dúvida em relação à conduta expectante é saber se os casos de obstrução tubária à HSG são prévios à GTI, ou seqüelas dela.

O indice de nova gravidez após conduta expectante, entre as pacientes que desejam conceber, é estimado entre 50 a $89 \%$. Em trabalho relativo à conduta expectante na GTI, Fernandez et al. ${ }^{3}$ mostraram que após 52 meses de seguimento, oito pacientes, dentre nove que desejavam engravidar, apresentaram gravidez normal. Não houve recidiva de GE. Uma paciente evoluiu para abortamento após 19 semanas de amenorréia. De acordo com o estudo de Ylöstalo et al. ${ }^{7}$, mais representativo pelo número de casos, o indice de nova gravidez estaria em torno de $60 \%$, incluindo-se todo o grupo tratado previamente, infértil ou não.

Pode-se concluir que a conduta expectante da GTI permite manter a anatomia tubária, apresenta baixa recidiva e possibilita um índice satisfatório de novas gestações intra-uterinas.

\section{SUMMARY}

Objective: to evaluate the inclusion parameters in the selection of cases of (tubal) ectopic pregnancy for expectant management and to assess the results. 
Methods: a prospective study was carried out in 70 patients with unruptured (tubal) ectopic pregnancy, with the objective to carry out an expectant management. The main inclusion criteria in this study were the diameter of the tubal mass, that should be equal or inferior to $5,0 \mathrm{~cm}$, reduced titles of beta-hCG (beta fraction of the chorionic gonadotropic hormone) as compared to the initial value within an interval of $48 \mathrm{~h}$, hemodynamic stability, wishes for future pregnancy and a written permission to participate in the study. All patients were observed in the hospital and when reduction in beta-hCG titles was observed, the patients were discharged from the hospital and followed in the outpatient department, with weekly determinations of beta-hCG until levels lower than $5 \mathrm{mIU} / \mathrm{ml}$ were reached, that were considered successful. Results: of the 70 patients who underwent expectant management, only one needed a surgical intervention, because of tubal rupture. The initial values of beta-hCG of the patients ranged from $27 \mathrm{mIU} / \mathrm{ml}$ to $41,000 \mathrm{mIU} / \mathrm{ml}$. The average diameter of the tubal mass was $2.9 \mathrm{~cm}$. The presence of free liquid in the peritoneal cavity was observed in 50 patients, small amount in 26 patients, moderate in 16 and large in 8 patients. The ultrasonographic resolution of hematosalpinx occurred in 58 patients and tubal ring was visualized in 12 patients. On color Doppler, 52 were at low risk and 18 at medium risk.

Conclusions: the expectant management should be applied with safety in the cases that respect the inclusion criteria, the index of success of this study being $98.6 \%$.

KEY WORDS: Ectopic pregnancy. Unruptured (tubal) ectopic pregnancy. Expectant management. Chorionic gonadotropic hormone.

\section{Referências:}

1. Lund JJ. Early ectopic pregnancy treated nonsurgically. J Obstet Gynaecol Br Empire 1955; 62:70-6.
2. Creinin MD, Washington AE. Cost of ectopic pregnancy management: surgery versus methotrexate. Fertil Steril 1993; 60:963-9.

3. Fernandez H, Rainhorn JD, Papiernik E, Bellet D, Frydman R. Spontaneous resolution of ectopic pregnancy. Obstet Gynecol 1988; 71:171-4.

4. Garcia AJ, Aubert JM, Sama J, Josimovich JB. Expectant management of presumed ectopic pregnancies. Fertil Steril 1987; 48:395-400.

5. Shalev E, Peleg D, Tsabari A, Romano S, Buatan M. Spontaneous resolution of ectopic pregnancy: natural history. Fertil Steril 1995; 63:15-9.

6. Trio D, Strobelt N, Picciolo C, Lapinski RH, Ghidini A. Prognostic factors of successful expectant management of ectopic pregnancy. Fertil Steril 1995; 63:469-72.

7. Ylöstalo P, Cacciatore B, Koskimies A, Kääriäinen M, Lehovirta $P$, Mäkelä $P$, et al. Conservative treatment of ectopic pregnancy. Ann N Y Acad Sci 1993; 626:516-23.

8. Elito Junior J. Índice orientador do tratamento sistêmico da prenhez ectópica íntegra com metotrexato em dose única $\left(50 \mathrm{mg} / \mathrm{m}^{2}\right)$, por via intramuscular. [Tese]. São Paulo: Escola Paulista de Medicina; 1997

9. Nyberg DA, Hughes MP, Mack LA, Wang KY. Extrauterine findings of ectopic pregnancy at transvaginal ultrasound: importance of echogenic fluid. Radiology 1991; 178: 823-6.

10.Atri M, Bret PM, Tulandi T. Spontaneous resolution of ectopic pregnancy: initial appearence and evolution at transvaginal US. Radiology 1993; 186: 83-6.

11.Sokal RR, Rohlf FJ. Biometry. San Francisco: W. H. Freeman; 1969. p. 776.

12.Pellerito JS, Taylor KJW, Quedens-Case C, Hammers LW, Scoutt LM, Ramos IM, et al. Ectopic pregnancy: evaluation with endovaginal color flow imaging. Radiology 1992; 183: 407-11.

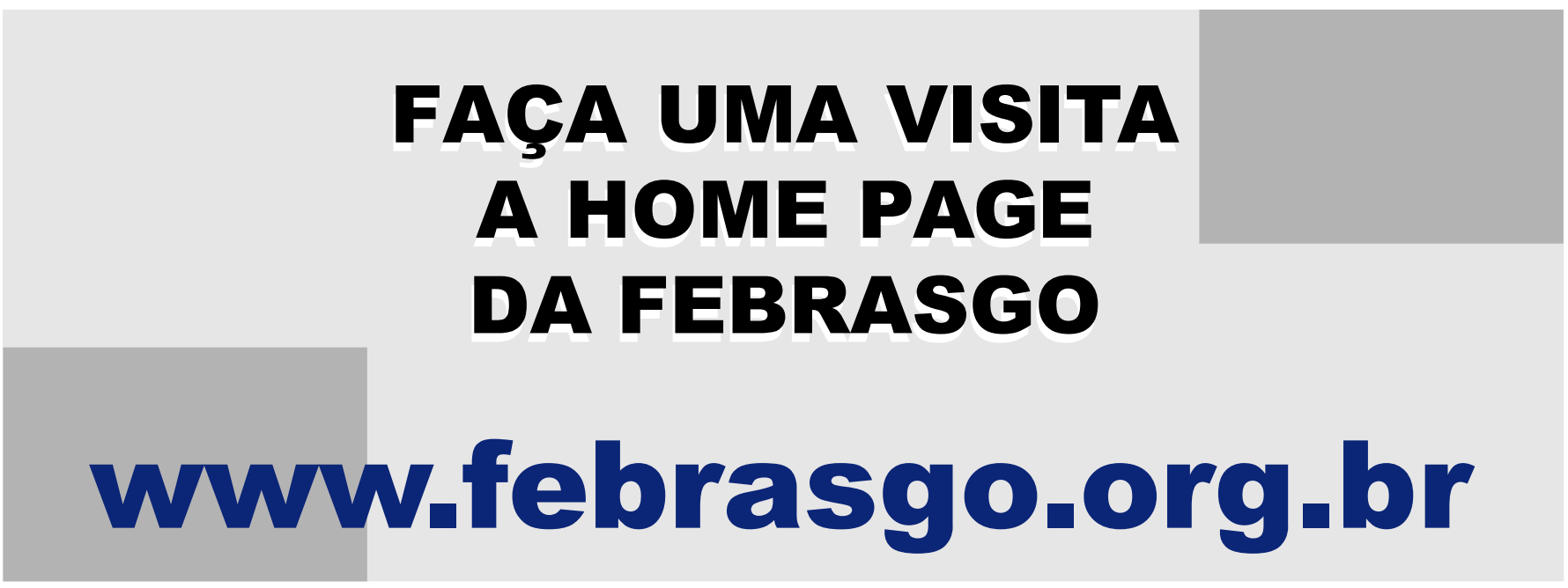

